\title{
Biotechnologies Applied to Intracranial Aneurysms \\ REVINW
}

\section{Patricia Bozzetto Ambrosi ${ }^{1}{ }^{3}$, Maria Tereza dos Santos Correia ${ }^{3}$, Laurent Spelle ${ }^{1}$, Jacques Moret ${ }^{1}$, Marcelo Moraes Valença ${ }^{2,3}$}

\section{Abstract}

Background: Despite several ongoing studies about new biotechnologies applied to intracranial aneurysms, particularly its genesis, its haemodynamic and its endovascular management; there are few studies with clinical validation until now.

Objective: To systematically review studies about biotechnologies used for the treatment of intracranial aneurysms.

Method: The authors searched the following databases: MEDLINE via PubMed (May 2014), EMBASE (May 2014), Science Citation Index Expanded (May 2014), AMED Allied Medicine (May 2014). It was considered for inclusion all studies when the role of biotechnologies and intracranial aneurysms were analysed using correlational or experimental researches.

Results: Three hundred fifty seven articles met the inclusion criteria. Most of the studies were about devices, flow studies, focused on bioinformatics, or computer science. The number of such investigations have proliferated considerably during the past five years.

Conclusion: Use of biotechnologies has raised expectations for both understanding of genesis and development of intracranial aneurysms and their strategical management. Based on current evidences, mostly small pilot trials, there are too few validated data. Refinements have dramatically improved flow studies in clinical settings.
1 Neuri- Beaujon, Interventional Neuroradiology Department, Clichy, France.

2 Neurosurgery Unit, Department of Neuropsychiatry, Federal University of Pernambuco, Recife, Brazil.

3 Postgraduate Program in Biological Sciences, Federal University of Pernambuco, Recife, Brazil.

Contact information:

Patricia Bozzetto Ambrosi.

Neuri; Hôpital Beaujon.

Address: 100 Boulevard du Général Leclerc; Clichy; 92110- France.

झ patty.bozzetto@gmail.com

\section{Keywords}

Intracranial Aneurysms; Flow; Bioinformatics; Biotechnology; Biotechnologies; Medical Devices.

\section{Introduction}

In the last years, biotechnologies - particularly intravascular flow modifiers- have been incorporated into the clinical management of intracranial aneurysm as a viable alternative to classical neurosurgery. 
[1-5] In the interim, many other biotechnological tools have been created to support and assist in interventional neuroradiology strategies. [6-8] These advances have been the subject of several studies, mostly clinical or experimental researches of intracranial aneuryms. [5-19] Biotechnologies applied to intracranial aneurysms encompass a number of tools and elements, including new techniques of treatment, computational flow dynamics (CFD) software programs to study flow, and updated strategies that may be used during the management of intracranial aneurysms. Unfortunately, however, many might not be routinely available in all catheterization laboratories. [7-12, 15-17]

Since measuring haemodynamic values directly in patients entails risk, models of patient-specific conditions have been used. The ultimate focus of these techniques is to mathematically describe and project the haemodynamic milieu of a diseased arterial wall. In turn, such models may be used to create automated algorithms, collectively surrogates, that predict focal weakening. Such analyses enable discrimination of aneurysmal risk, thereby guiding treatment decisions.

Enthusiasm generated by these studies should be tempered by the paucity of compelling data demonstrating tangible clinical benefits this new biotechnological arsenal. [5-19] Systematic reviews on the role of biotechnologies and intracranial aneurysms are expected. The aim of this study was to systematically review biotechnology studies concerning including new devices, flow investigations, and/ or bioinformatics as applied to the management of intracranial aneurysms. [6-7]

\section{Methods}

\section{Eligiblity Criteria}

The review was based upon data from clinical and research studies about biotechnologies and intracranial aneurysms. Studies in children and those in conventional MR imaging were excluded. Additional search about carotid aneurysm, carotid siphon and aneurysms classification was included but it were not considered in the final analysis.

\section{Search Strategy}

The authors searched MEDLINE via PubMed (May 2014), EMBASE (May 2014), Science Citation Index Expanded (May 2014) and AMED Allied Medicine (May 2014). A set of specific terms were used to devise the search strategy described in Table 1. The initial strategy was to match single words, then combine terms, their derivatives, and related terms. Thesaurus, MeSH and systematization of bibliographic searches were added in order to improve the search. Only articles written in English were included.

Table 1. Search strategy.

Results

Medline

1. (intracranial and aneurysm).ti,ab,af;

24026

2. biotechnologies.ti,ab, af

3847

3. biotechnology.ti,ab,af

212594

4. flow.ti,ab,af

601190

5. exp biotechnology/

49069

6. exp *biotechnology/

29366

7. exp biotechnology/

49069

8. 1 and 3 and 4

9. 1 and 4

2506

10. (intracranial and aneurysm).ti,ab

6888

11. (intracranial and aneurysm).ti,ab,af

24026

12. flow.ti,ab,af

601190

13. biotechnology.ti,ab,af

212594

14. bioinformatics.ti,ab, af

47648

15. biorobotics.ti,ab, af

186

16. (computer and science).ti,ab,af

60145

17. devices.ti,ab

110299

18. 2 and 3 
19. 2 and 4

Results

20. 2 and 5

12. 2 and 7

13.2 and 8

303

14. 13 [limit to: (publication types clinical trial, all or clinical trial, phase I or clinical trial, phase II or clinical trial, phase III or clinical trial, phase IV or clinical trial)]

15. 2 and 3 and 7 and 8

0

17. 7 and 8

18. 2 and 17

19. model.ti,ab [limit to: (publication types clinical trial, all or clinical trial, phase I or clinical trial, phase II or clinical trial, phase III or clinical trial, phase IV or clinical trial)]

20. 2 and 3 and 19 [limit to: (publication types clinical trial, all or clinical trial, phase I or clinical trial, phase II or clinical trial, phase III or clinical trial, phase IV or clinical trial)]

21. 20 [limit to: (publication types clinical trial, all or clinical trial, phase I or clinical trial, phase II or clinical trial, phase III or clinical trial, phase IV or clinical trial)]

22. (optic and flow).ti,ab,af

23. 2 and 22

24. 9 [limit to: (publication types clinical trial, all or clinical trial, phase I or clinical trial, phase II or clinical trial, phase III or clinical trial, phase IV or clinical trial)]

Embase

25. (intracranial and aneurysms).ti,ab,af

10990

26. flow.ti,ab,af

768434

25 and 26

1527

26. (carotid and aneurysm).ti,ab

27. classification.ti,ab,af

501997

28. 25 and 29

318

Medline

29. (carotid and siphon).ti,ab, af [limit to: (publication types clinical trial, all or clinical trial, phase I or clinical trial, phase II or 15 clinical trial, phase III or clinical trial, phase IV or clinical trial)]

\section{Data analysis}

The data extracted were (a) year of publication; (b) number of subjects; (c) the type of methods used to assess flow as experimental, computational or others; (d) whether it was a clinical or research study; (e) whether it concerned genesis of aneurysms; (f) treatment; (g) prevention; (h) the clinical impact of the study in routine clinical practice; and/or (i) whether the study employed vascular ultrasound.

\section{Results}

\section{Search Results}

A total of 24026 publications about intracranial aneurysms, 212594 publications about biotechnology, 601190 publications about flow, and 2056 publications about both intracranial aneurysms and flow were found.

There were 357 articles identified that focused upon biotechnologies (flow, biotechnology, bioinformatics, biorobotics, computer and science, devices) as described in Figure 1. Using the strategy of inclusion of Publication Types Clinical Trial, a relative lack of validated studies about devices and intracranial aneurysms became apparent. Flow, biotechnology, bioinformatics, biorobotics, computer and science were added to improve our search. The majority of studies were limited to case reports and small pilot series.

In seeking to broaden the spectrum analysis, we added the words model and optic flow, by referring to the experimental models, respectively only 6 and 13 studies were identified, following the words flow and intracranial aneurysms were matched and 128 results were founded.

All told, 22 studies met the basic inclusion criteria -biotechnologies versus intracranial aneurysms- and were subsequently filtered by reading, and were clinical trials, Phase I or Phase II identified (Figure 1). 
Figure 1: Flow of elements and filters used to retrieve final studies included..
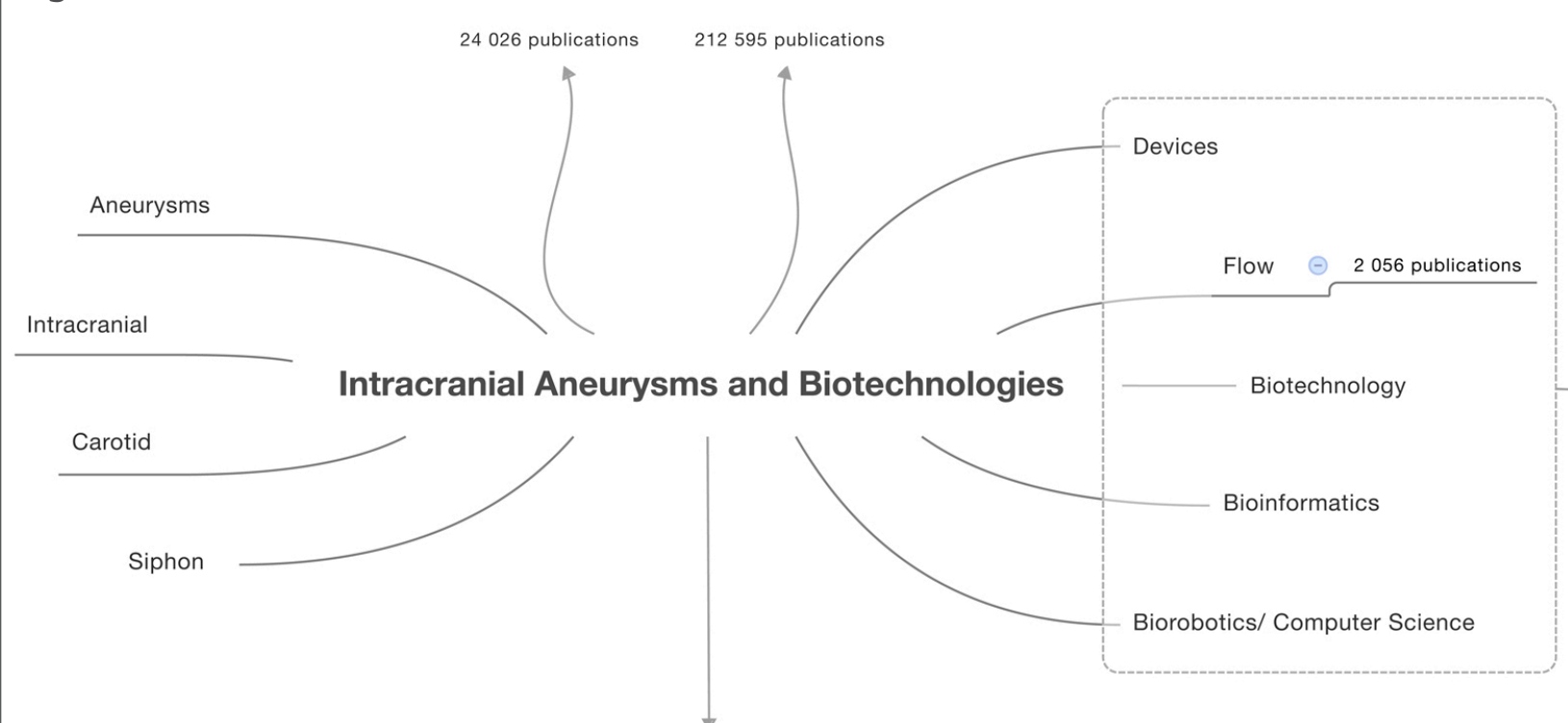

\section{Subgroup Analyses and Clinical Applications}

Experimental studies described were in vitro vascular models and in vivo vascular models developed in animals which were used to analyze the dynamic flow within intracranial aneurysms induced by flow diverters studying flow effects, thrombosis and endothelialization after flow diversion. Computational methods has been an improvement over the conventional angiographical studies. Additional technologies have been added using angiography and/or other dynamic methods.

Other clinical applications include the identification and stratification of rupture risk in aneurysms, which might lead to detect possible future preventive treatment.

\section{Discussion}

This brief review found that new biotechnologies have been incorporated into the management of intracranial aneurysms in proportion to the volume of published research. However, the validity of these tools in clinical management, ie, outcomes, remains to be shown. [1-5, 6-9]
Flow is believed to be a major factor involved in many steps during the natural history of intracranial aneurysms, including initiation, growth, degeneration, rupture, and recurrence recanalization. Due to the complex modeling of patient-specific aneurysms, it has been important to validate new technologies to better understand the pattern of aneurysmal flow. [6-9, 14-20]

With the improvement of computer power and evolution in CFD, numerical simulations gained in speed and precision. Numerical simulations may include assessment of effects upon flow within the aneurysm and flow within the parent artery and its branches. [9, 16-17] Quantifiable flow values of velocities and pressure within the virtual aneurysm are compared with their related virtual parent artery parameters including velocity, helicity (rotation), wall shear stress and oscillatory shear index, among others. Many haemodynamic variables, including flow pattern and wall shear stress, are hypothesized to be the causes of growth and development of intracranial aneurysms. [9, 15, 16]

Blood flow simulation of intracranial aneurysms in patient-specific aneurysms according to specific 
geometries, presence of hypertension, or whether an aneurysm has been stented or otherwise treated is now considered an older approach. In the past decade, CFD has evolved to become much easier, faster, and less expensive to use. The combination of advanced image processing and geometrical modeling techniques with computational fluid dynamics enhanced the generation of detailed haemodynamic aneurysmal flow in patient-specific geometries. [5-6, 9, 10, 13]

Flow measurements help understand the mechanisms that influence the intracranial aneurysmal wall and therefore its biology during initiation, growth, degeneration, inflammation, and regrowth. Flow data may be assessed individual or together. Assessing flow dynamics in vitro vascular phantoms is done to estimate the in vivo flow changes that might be expected. $[9,18]$ Even though CFD has become more management on a practical basis, controversy persists over the need to incorporate patient-specific conditions into computational analysis. [5-6, 10]

Aneurysm model flow measurements are used to define inflow and outflow boundary conditions for computational haemodynamic models of the aneurysms. The additional in situ measurements which are not recorded in the simulation are then used to assess the accuracy of the simulated flow velocity and pressure drop. Simulated velocities using patient-specific boundary conditions show good agreement with the guidewire measurements at measurement locations inside the domain, with no bias in the agreement and a random scatter of $25 \%$. Simulated velocities using the simplified, literature-derived values show a systematic bias and over-predicted velocity by $30 \%$ with a random scatter of $40 \%$. [5-6]

Computational haemodynamics using endovascularly measured patient-specific boundary conditions have the potential to improve treatment predictions as they provide more accurate and precise results of the aneurysmal haemodyna- mics than those based on commonly accepted reference values for boundary conditions. $[6,10]$ Recent development of computational tools has allowed the study of patient aneurysm geometry in actual patients through model reconstruction. [9, 17-19]

Despite these limitations, magnetic resonance angiography (MRA) images could provide realistic quantifications of the volumetric blood flow rates along the major cerebral vessels and visualizations of the main blood flow structures, and can play an important role in the assessment of haemodynamic disturbances in patients. [20]

The limited image resolution means that some of the smaller vessels such as the ophthalmic artery or some of the communicating arteries may not be detected in the MRA images. However, the spatial and temporal resolutions of the phase-contrast magnetic resonance (PC-MR) images can be increased at the cost of scanning time.

\section{Conclusion}

Despite the great technologies have emerged from men validated discoveries. Based on current evidences, the role of biotechnologies versus management of intracranial aneurysms had been studied by small pilot trials. The heterogeneity of studies makes them difficult to compare variations in selection and methods. Further studies with clinical strong theoretical basis and standardization will be essential to recognize biotechnologies as new tool to management of intracranial aneurysms. Technically, researchers are now able to reproduce flow dynamics using specific modeling of aneurysms. This ability will lead to significant changes in decision-making process of management of intracranial aneurysms in the near future. 


\section{Acknowlegments}

Thanks so much to the library's staff of the Lancashire Teaching Hospital NHS Foundation Trust (United Kindgom) for helping us with the systematic review.

I deeply appreciate the support of Prof. Dr. Vasconcelos, C.A.C. (Department of Nutrition, UFPE, Recife, Brazil) for his help in the finalization of this this manuscript and other suggestions.

\section{Disclosures}

P.B.A received a scholarship grant funded by Capes Education Bursary, Ministry of Education, Brazil at Beaujon University Hospital, Clichy and University Paris Diderot, Paris, France for her phD.

\section{References}

1. Nelson PK, Lylyk P, Szikora I, Wetzel SG, Wanke I, Fiorella D. The pipeline embolization device for the intracranial treatment of aneurysms trial. Am J Neurorad 2011; 32(1): 34-40.

2. Medical University of South Carolina. LARGE aneurysm randomized trial: flow diversion versus traditional endovascular coiling therapy, in ClinicalTrials.Gov, NCT01762137, National Library of Medicine (US), Bethesda, MD, USA, 2000, http://clinicaltrials.gov/ct2/show/record/NCT01762137. Accessed on July 19, 2015.

3. The International Study of Unruptured Intracranial Aneurysms Investigators: Unruptured intracranial aneurysms: natural history, clinical outcome, and risks of surgical and endovascular treatment. Lancet 2003; 362: 103-110.

4. Williams $L N$, Brown RD. Management of unruptured intracranial aneurysms. Neurol Clin Pract 2013; 3(2): 99-108.
5. Cebral JR, Castro MA, Appanaboyina S, Putman CM, Millan D, Frangi AF. Efficient pipeline for image-based patient specific analysis of cerebral aneurysm haemodynamics: technique and sensitivity. IEEE Trans Med Imaging 2005; 24: 457-67.

6. Sun Q, Groth A, Aach T Comprehensive validation of computational fluid dynamics simulations of in-vivo blood flow in patient-specific cerebral aneurysms. Med Phys. 2012; 39(2): 742-54. doi: 10.1118/1.3675402.

7. Levit M, McGah PM, Aliseda A, Mourad PD, Nerva JD, Vaidya SS, Morton RP, Ghodke BV, Kim LJ. Cerebral aneurysms treated with flow-diverting stents: computational models using intravascular blood flow measurements. Am J Neuroradiol 2014; 35(1): 143148.

8. Jeong W, Rhee K. Haemodynamics of cerebral aneurysm: computational analyses of aneurysm progresss and treatment. Comput Math Methods Med 2012; 782-801. doi: 10.1155/2012/782801. Epub 2012 Feb 19

9. Ausgburger L, Reymond P, Fonck E, kulcsar Z, Farthat M, Ohta M, Stergiopulos N, Rufenacht DA. Methodologies to assess blood flow in cerebral aneurisms: current state of state and perspectives. J Neuroradiol 2009; 36(5): 270-277.

10. Valen-Sendstad K, Steinman DA. Mind the gap: impact of computational fluid dynamics solution strategy on prediction on intracranial aneurysm haemodynamics and rupture status indicators. Am J Neuroradiol 2014; 35(3): 536-43.

11. Aenis $M$, Stancampiano AP et al. Modeling of flow in a straight stented and nonstented side wall aneurysm model. Journal of Biomechanical Engineering-Transactions of the ASME 1997; 119(2): 206-212.

12. Wang W, Graziano F, Russo V, Ulm AJ, De Kee D, Khismatullin DB. Giant intracranial aneurysm embolization with a yield stress fluid material: insights from CFD analysis. Biorheology. 2013; 50(3-4): 99-114

13. Watton PN, Raberger NB, Holzapfel GA, Ventikos Y. Coupling the haemodynamic environment to the evolution of cerebral aneurysms: computational framework and numerical examples. J Biomech Eng 2009; 131(10): 101003

14. Weichert F, Walczak L, Fisseler D, Opfermann T, Razzaq M, Münster $\mathrm{R}$, et al. Simulation of intra-aneurysmal blood flow by different numerical methods. Comput Math Methods Med. 2013.

15. Wong GK, Poon WS. Current status of computational fluid dynamics for cerebral aneurysms: the clinician's perspective. J Clin Neurosci 2011; 18(10): 1285-8.

16. Wu C, Xu BN, Sun ZH, Wang FY, Liu L, Zhang XJ, et al. Different treatment modalities of fusiform basilar trunk aneurysm: study on computational haemodynamics. Chin Med J (Engl) 2012; 125(1): 97-101. 
17. Steinman DA, Milner JS, Norley CJ, Lownie SP, Holdsworth Image-based computational simulation of flow dynamics in a giant intracranial aneurysm. Am J Neurorad 2003; 24: 55966

18. Cebral JR, Castro MA, Burgess JE, Pergolizzi RS, Sheridan MJ, Putman CM. Characterization of cerebral aneurysms for assessing risk of rupture by using patient-specific computational haemodynamics models. Am J Neuroradio 2005; 26: 2550-59.

19. Cebral JR, Sheridan M, Putman CM. 2s and bleb formation in intracranial aneurysms. Am J Neuroradiol 2010; 31: 304-10.

20. Rayz VL, Boussel L, Acevedo-Bolton G, Martin AJ, Young WL, Lawton MT, Higashida R, Saloner D. Numerical simulations of flow in cerebral aneurysms: comparison of CFD results and in vivo MRI measurements. J Biomech Eng. 2008; 130(5): 051011. doi: 10.1115/1.2970056

\section{Comment on this article:}

\section{$f(B)$ in $8+S$ P}

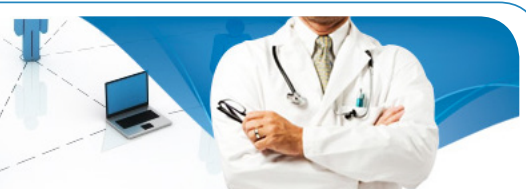

\section{http://medicalia.org/}

Where Doctors exchange clinical experiences, review their cases and share clinical knowledge. You can also access lots of medical publications for free. Join Now!

\section{Publish with iMedPub}

\section{http://www.imed.pub}

International Archives of Medicine is an open access journal publishing articles encompassing all aspects of medical science and clinical practice. IAM is considered a megajournal with independent sections on all areas of medicine. IAM is a really international journal with authors and board members from all around the world. The journal is widely indexed and classified Q1 in category Medicine. 